\title{
Effective Antitachycardia Therapy by Temporary External Defibrillator Utilized as Bridge to Reimplantation
}

\author{
Giulio Falasconi ${ }^{1}$, Giuseppe d'Angelo ${ }^{2}$, Luigi Pannone ${ }^{2}$, Alessandra Marzi ${ }^{2}$, and Patrizio \\ Mazzone $^{2}$ \\ ${ }^{1}$ San Raffaele Hospital \\ ${ }^{2}$ Ospedale San Raffaele
}

October 5, 2020

\begin{abstract}
The increasing number of Cardiac Implantable Electronic Device (CIED)-carriers is leading to a growing need of transvenous lead extraction of various levels of complexity. Although still little utilized for patients with CIED-related infection and concomitant history of VT, report a peculiar case of transvenous lead extraction with the following temporary external cardioverter-defibrillator implantation as a bridge to reimplantation; this bridge system had a life-saving role for the patient by stopping an episode of sustained ventricular tachycardia through antitachycardia pacing.
\end{abstract}

\section{Effective Antitachycardia Therapy by Temporary External Defibrillator Utilized as Bridge to Reimplantation}

Giulio Falasconi $\mathrm{MD}^{1}$, Giuseppe D'Angelo $\mathrm{MD}^{2 *}$, Luigi Pannone $\mathrm{MD}^{1}$, Alessandra Marzi $\mathrm{MD}^{2}$, Patrizio Mazzone $\mathrm{MD}^{2}$

1. Clinical Cardiology Unit, IRCCS San Raffaele Hospital and Vita-Salute University, Milan, Italy.

2. Department of Cardiac Electrophysiology and Arrhythmology, IRCCS San Raffaele Hospital and VitaSalute University, Milan, Italy.

Conflicts of interest: none of the authors have any competing interests.

The author received no specific funding for this work.

The authors have reported that they have no relationships relevant to the contents of this paper to disclose.

*Corresponding author:

Giuseppe D'angelo, MD

IRCCS San Raffaele University Hospital, Milan, Italy

Tel. 003928502344

Email: dangelo.giuseppe@hsr.it

Keywords: antitachycardia pacing, Implantable Cardioverter Defibrillator, pocket infection, transvenous extraction, ventricular tachycardia.

Abstract:

The increasing number of Cardiac Implantable Electronic Device (CIED)-carriers is leading to a growing need of transvenous lead extraction of various levels of complexity. Although still little utilized for patients with 
CIED-related infection and concomitant history of VT, report a peculiar case of transvenous lead extraction with the following temporary external cardioverter-defibrillator implantation as a bridge to reimplantation; this bridge system had a life-saving role for the patient by stopping an episode of sustained ventricular tachycardia through antitachycardia pacing.

Manuscript:

A 52-year-old man presented for Cardiac Implantable Electronic Device (CIED) pocket infection (Figure A ). His past medical history was significant for mitral valve repair plus annuloplasty with complete ring complicated by periprocedural myocardial infarction, followed by bicameral Implantable Cardioverter Defibrillator (ICD) implantation in primary prevention. After two uneventful years, one month before the index hospitalization, the patient experienced a first appropriate ICD shock subsequent to ATP failure for ventricular tachycardia (VT). During the hospitalization multiple appropriate ICD interventions for VT were detected.

The patient underwent to a single-step procedure of generator removal and complete transvenous extraction of both leads, performed according to our hospital stepwise approach by traction on a locking stylet (Lead Locking Device ${ }^{\mathrm{TM}}$, Spectranetics ${ }^{\circledR}$ ) with contextual implantation of a bridge system with an active-fixation dual-coil lead (Durata $^{\text {TM }}$, St. Jude Medical) (Figure B, Video 1, Video 2 ). This system was inserted through percutaneous puncture of left subclavian vein, attached to theskin with spaced silk stitches; finally, the lead was connected to the generator disinfected and fixed externally (Figure C, D ). The previous programming of the VT therapy was maintained, utilizing the dual-coil ICD lead to provide an effective shocking vector.

One week later, the patient developed one episode of sustained VT, interrupted by a second burst of antitachycardia pacing (Figure E).

Blood cultures at the admission and two days after extraction were both negative and the patient was started on daptomycin plus ceftriaxone after Staphylococcus Warneri isolation from catheter tips culture. A contralateral ICD was implanted after 15 days of specific antibiotic therapy and the remaining hospital stay was uneventful.

The increasing number of CIED-carriers is leading to a growing need of transvenous lead extraction (TLE) of various levels of complexity. Although still little utilized for patients with CIED-related infection and concomitant history of VT, TLE with the following temporary external cardioverter-defibrillator implantation as a bridge to reimplantation is a safe and effective possibility

\section{FIGURE LEGEND}

Figure A . Cardiac Implantable Electronic Device pocket infection.

Figure B . Fluoroscopic image of the implantation of a bridge system with an active-fixation dual-coil lead (Durata $^{\mathrm{TM}}$, St. Jude Medical).

Figure C . Patient's generator (Boston Scientific) disinfected and fixed externally.

Figure D . Post-procedural Radiography.

Figure E . Episode of sustained VT, interrupted by a second burst of antitachycardia pacing. 


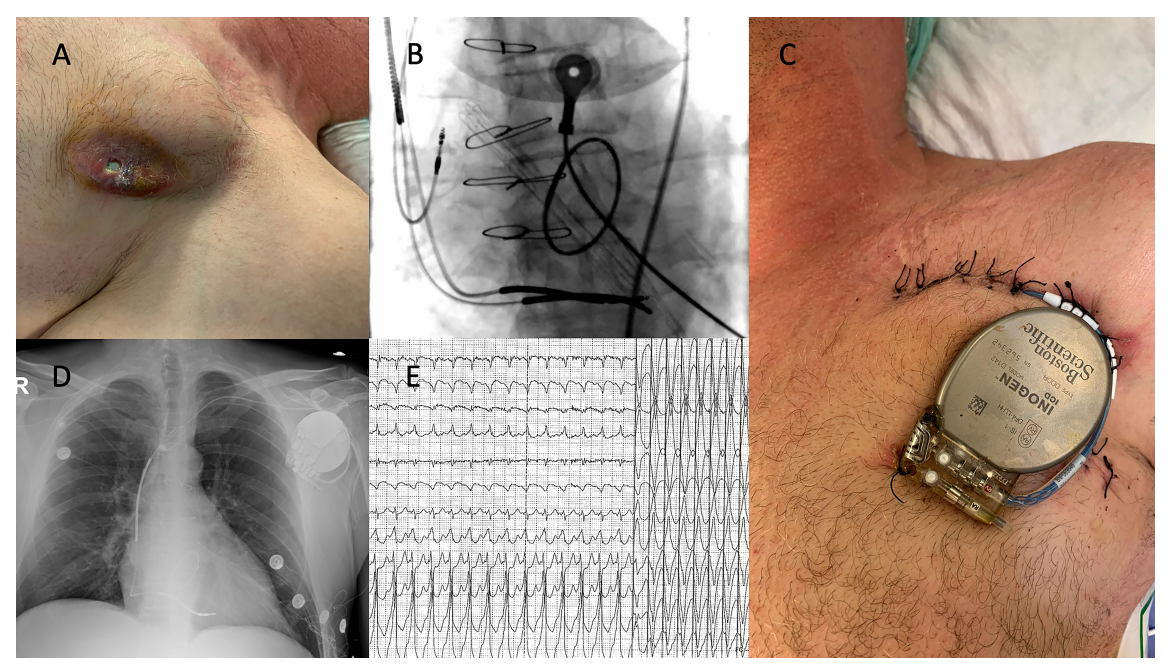

